\title{
Digital Imaging for the Education of Proper Surgical Hand Disinfection ${ }^{\star}$
}

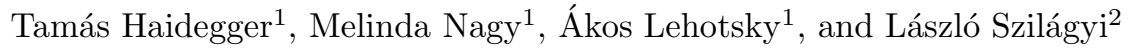 \\ 1 Budapest University of Technology and Economics, Dept. of Control Engineering \\ and Information Technology, Budapest, Hungary \\ haidegger@iit.bme.hu, meliole28@gmail.com, akos.lehotsky@gmail.com \\ 2 Sapientia - Hungarian Science University of Transylvania, \\ Faculty of Technical and Human Science, Tîrgu-Mureş, Romania \\ lalo@ms.sapientia.ro
}

\begin{abstract}
Nosocomial infections are the undesirable result of a treatment in a hospital, or a health care service unit, not related to the patient's original condition. Despite the evolution of medicine, fundamental problems with hand hygiene remain existent, leading to the spread of nosocomial infections. Our group has been working on a generic solution to provide a method and apparatus to teach and verify proper hand disinfection. The general idea is to mark the skin surfaces that were sufficiently treated with alcoholic hand rub. Digital image processing is employed to determine the location of these areas and overlay it on the segmented hand, visualizing the results in an intuitive form. A non-disruptive ultraviolet marker is mixed to a commercially available hand rub, therefore leaving the original hand washing workflow intact. Digital images are taken in an enclosed device we developed for this purpose. First, robust hand contour segmentation is performed, then a histogram-based formulation of the fuzzy $c$-means algorithm is employed for the classification of clean versus dirty regions, minimizing the processing time of the images. The method and device have been tested in 3 hospitals in Hungary, Romania and Singapore, on surgeons, residents, medical students and nurses. A health care professional verified the results of the segmentation, since no gold standard is available for the recorded human cases. We were able to identify the hand boundaries correctly in $99.2 \%$ of the cases. The device can give objective feedback to medical students and staff to develop and maintain proper hand disinfection practice.
\end{abstract}

Keywords: surgical hand disinfection, hospital acquired infection, image segmentation, semi-supervised fuzzy c-means clustering.

\footnotetext{
* This work was supported in part by Contact Singapore, the Austrian Center for Medical Innovation and Technology and the Hungarian national grants NKTH OTKA CK80316 and TÁMOP-4.2.1/B-09/1/KMR-2010-0002. The work of L. Szilágyi was supported by CNCSIS-UEFISCSU, project no. PD_667, contract no. 28/05.08.2010. 


\section{Introduction}

Ignaz Semmelweis realized first the importance of hand hygiene in the medical environment in 1847, and imposed strict regulations in his hospital, immediately resulting in better patient survival rates. While medical technology has tremendously improved along the years, it has failed to eliminate the persistent problem of incomplete hand hygiene, leading to the spread of nosocomial infections. Infections are considered nosocomial - also referred to as Hospital-Acquired Infections (HAI) - if they appear 48 hours or more after hospital admission or within 30 days after discharge. HAI occurrence has been reported in $7.1 \%$ on average in the Western world [1. Sadly, HAI is responsible for 15,000 deaths in Europe and over 90,000 world wide every year [2]. In addition, it causes $\$ 2 \mathrm{~B}$ extra cost annually in the UK [3, \$4.5-5.7B direct 4 and $\$ 17-40 \mathrm{~B}$ additional costs in the USA, as it may triple average hospitalization time [35.

The pathogens are most often transferred via the hands of the medical staff, therefore compliance with the operative hand hygiene protocols and standards is absolutely necessary [6/78]. Fig. 1(a) shows the most frequently missed parts of the hands based on a multi-center study conducted by the US Center of Disease Control and Prevention (CDC) 9]. Reports suggest that accurate hand disinfection could reduce HAI by up to $30 \%$, saving hundreds of lives each month and reducing hospitalization costs significantly [10]11.

In this paper, a novel approach to hand disinfection education and quality control is discussed. The employed workflow is very intuitive: disinfection is performed with a liquid, alcohol-based hand rub mixed with UV reflective powder, which appears brighter under adequate UV-C lighting (on surfaces having sufficiently treated with the soap). Pictures of the hands are taken in a UV lighted case (users should spread their fingers to provide good visual access), after which a regular notebook performs the evaluation. It provides graphical results (highlighting clean versus dirty areas), and indicates an overall quantitative score. We can obtain objective, measurable and scalable data on hand disinfection quality, that is most useful to train medical students to acquire good hand washing practice.

\section{Methods}

\subsection{Image Recording}

A compact, mobile device was built for comparable recording and objective assessment of hand disinfection quality, shown in Fig. 1(b). This consists of removable plastic plates, making it light and transportable. Dimensions are $35 \times$ $30 \times 30 \mathrm{~cm}(\mathrm{w} / \mathrm{l} / \mathrm{h})$ providing dual hand imaging option. There are 21 lines with $10 \mathrm{UV}$ LEDs in each row, fixed on the top plate. LEDs provide equal illumination on the hand surface. The current is minimized to $30 \mathrm{~mA}$, therefore any USB port is sufficient for power supply, not constraining the mobility of the device. In the center of the cover, a digital camera (Canon PowerShot SX100) is placed, controlled through the connected laptop. The whole structure is covered 

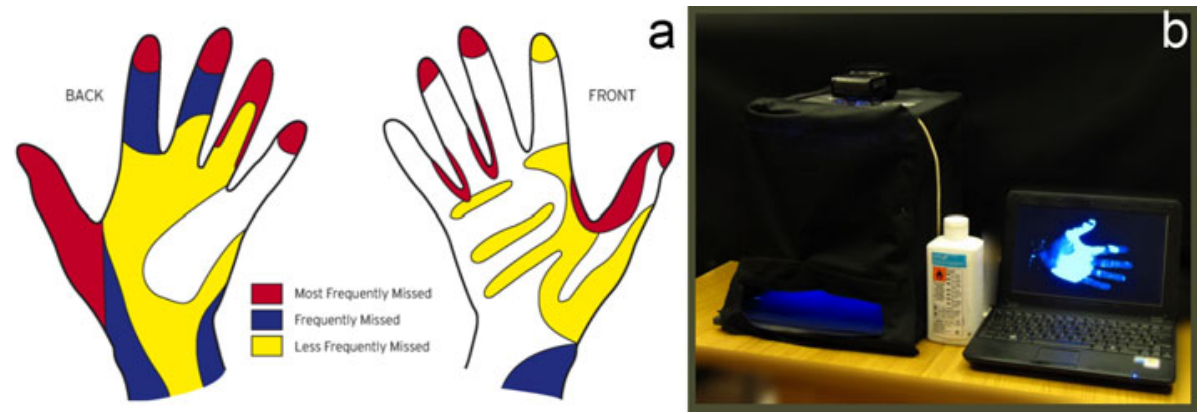

Fig. 1. (a) Results of a study by CDC, showing the most frequently missed parts of the hands after surgical hand disinfection, based on a US survey [9]; (b) Hardware setup for objective hand disinfection control

with removable drapes, reducing the disturbance of external light. The compact device was designed to be wall-mountable for upcoming prolonged (repetitive) clinical trials. In order to keep the interior of the box clean, we use disposable matte black paper covers.

\subsection{Initial Considerations for Image Processing}

The aim of the system is to provide an objective and repeatable measurement of hand washing through image segmentation and classification by determining the ratio of the clean (bright) surface compared to the whole skin surface. To be able to compute this, the Region of Interest (ROI) is first selected, separating the hand from the background. Next, the pixels within the ROI are fed to a semi-supervised classification process, to distinguish clean areas from dirty ones. Finally, the ratio of the clean areas over the whole hand is calculated.

For medical applicability, it is important to find a good compromise between processing speed and image resolution. Accordingly, captured images are resized to $400 \times 300$ pixels. Although the camera captures three intensity channels (red, green and blue), we restricted the procedure to use the green channel, since the red channel's intensity is very low in the reduced-light environment, therefore it rarely contains less information. On the other hand, during the initial trials, it was noticed that the blue channel is often saturated.

\subsection{ROI Selection}

The first step of the procedure mostly consists of image processing operations that can be easily fed to parallel execution:

1. A median filter of $5 \times 5$ mask is applied to get rid of isolated pixels containing high-frequency imaging artifacts. 
2. The complex morphological operation is applied to the filtered image:

$$
I_{g}=\left[\left(I_{f} \oplus b\right)-\left(I_{f} \ominus b\right)\right] \times f\left(I_{f} \ominus b\right),
$$

where $b$ is a $3 \times 3$ square shaped structuring element, $I_{f}$ stands for the filtered image, $I_{g}$ represents the computed gradient image, and $f(\cdot)$ is an amplification function. The role of $f(\cdot)$ is to amplify the edges that are situated in the proximity of dark pixels in the filtered image, and suppress other edges, which are supposed to be inside the area of the hand. $f(\cdot)$ is an exponentially decreasing function that crosses the unit value in a context dependent threshold intensity level.

3. In gradient image $I_{g}$, the background typically contains visible texture, while the inner area of the hand is uniformly dark. A region growing method is applied to identify the pixels belonging to the hand. In order to select seed points, a uniform $11 \times 11$ averaging mask is applied to the image $I_{g}$. Minimum intensity pixels of the smoothed image can be used as seed points.

4. Finally, the pixels belonging to the amplified contour of the hand are separated, in accordance with their original intensities.

The intermediary and final results of ROI selection are shown in Fig. 2(a)(f). After the ROI is established, the relative histogram of the inner pixels is computed, using the intensities of the filtered image, $I_{f}$. This histogram (see $p_{l}$ values in Section 2.4) will be the input data of the semi-supervised classification.

\subsection{Semi-supervised Classification}

To minimize the processing time of the images, a quick, histogram-based formulation [12] of the fuzzy $c$-means algorithm (FCM) [13] is employed for the separation of pixels belonging to clean and dirty regions of the hand. The main reason for this approach is its ability to classify intensity levels instead of pixels, resulting in high execution speed. Supervision is introduced into the objective function of FCM by the second additive term, and its strength is controlled by the supervision rate $\sigma$ that acts like a trade-off parameter between the unsupervised and supervised terms. The proposed objective function is:

$$
J_{\mathrm{ssFCM}}=(1-\sigma) \sum_{i=1}^{c} \sum_{l=1}^{q} p_{l} u_{i l}^{m}\left(l-v_{i}\right)^{2}+\sigma \sum_{i=1}^{c} \sum_{l=1}^{q} \widetilde{p}_{i l}\left(l-v_{i}\right)^{2},
$$

where $c$ represents the number of clusters, $q$ is the number of intensity levels in the input image, $v_{i}$ is the prototype intensity of cluster with index $i, u_{i l}$ is the fuzzy membership function representing the probability of gray shade $l$ to be assigned to cluster represented by $v_{i}$ and $m>1$ is the fuzzy exponent as defined in [13. Further on, $p_{l}$ is the percentage of pixels situated in the ROI having their intensity equal to $l$ and similarly, $\tilde{p}_{i l}, l=1 \ldots q$ describe the intensity distribution of the labeled data belonging to cluster index $i$. These previously labeled pixels were extracted from several expert-evaluated reference images, and they are assigned to clusters using hard (bivalent) partition. 
The objective function $J_{\mathrm{ssFCM}}$ is optimized under the probabilistic constraint $\sum_{i=1}^{c} u_{i l}=1, \forall l=1 \ldots q$. The optimum is reached by alternately optimizing along $u_{i l}$ with $v_{i}$ fixed and along $v_{i}$ with $u_{i l}$ fixed, until the cluster prototypes converge. The update formulas of fuzzy memberships and cluster prototypesobtained using zero gradient conditions and Lagrange multipliers - are:

$$
u_{i l}=\frac{\left(l-v_{i}\right)^{-2 /(m-1)}}{\sum_{j=1}^{c}\left(l-v_{j}\right)^{-2 /(m-1)}}, \quad \forall i=1 \ldots c, \forall l=1 \ldots q,
$$

and

$$
v_{i}=\frac{\sum_{l=1}^{q}\left[(1-\sigma) p_{l} u_{i l}^{m}+\sigma \widetilde{p}_{i l}\right] \times l}{\sum_{l=1}^{q}(1-\sigma) p_{l} u_{i l}^{m}+\sigma \widetilde{p}_{i l}}, \quad \forall i=1 \ldots c .
$$

Clustering is executed with the class count initially set to $c=3$. Besides the clean and dirty areas, an intermediate (so-called partially washed) area is also identified. However, depending on the final intensity values of the cluster prototypes, this middle cluster may be merged into the dirty or clean class, due to excessive proximity. In the next phase of the development, we intend to involve patient-specific data (skin color, vessels, birthmarks, etc.) to facilitate the final decision on the intermediate class.

\subsection{Interpretation of Classification}

At this stage, pixels are clustered regardless of their position within the ROI. Next, we apply certain context-dependent criteria to improve the prediction accuracy. A regularization filter is used to remove isolated pixels of any class, surrounded by another class. This also helps to remove small spots that originate from uneven skin color. Furthermore, a weighting factor is assigned to each pixel within the ROI (with lower weight to pixels situated along the edges), to minimize the influence of shadows on the final estimation. The weighted summation of correctly disinfected areas of the hand is achieved through:

1. A distance transform is employed to separate the pixels within the ROI into edge pixels and inner pixels. Edge pixels are situated within a close neighborhood of the non-hand regions. We denote by $n$ the number of edge pixels, while the number of inner pixels to be referred to as $N$.

2. We differentiate and count clean and dirty pixels within the set of edge pixels ( $n_{c}$ and $n_{d}$ will denote the count of clean and dirty edge pixels, respectively) and inner pixels ( $N_{c}$ and $N_{d}$, analogously).

3. The non-weighted estimation of the clean area is given as:

$$
\pi=100 \times \frac{N_{c}+n_{c}}{N_{c}+n_{c}+N_{d}+n_{d}} .
$$

4. The weighted (edge-compensated) percentage of the disinfected area is:

$$
\pi_{w}=100 \times \frac{N_{c}+\alpha n_{c}}{\left(N_{c}+N_{d}\right)+\alpha\left(n_{c}+n_{d}\right)} .
$$

5. In order to suppress shading artifacts, an $\alpha=0.2$ weighting factor is recommended. This value was established empirically. 


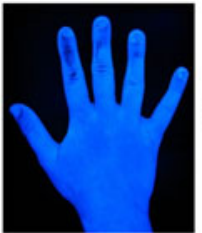

(a)

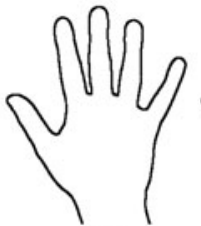

(d)

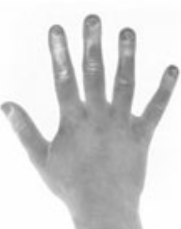

(b)

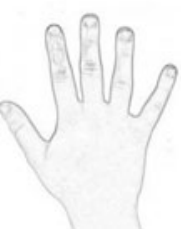

(c)

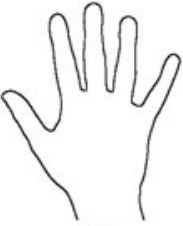

(e)

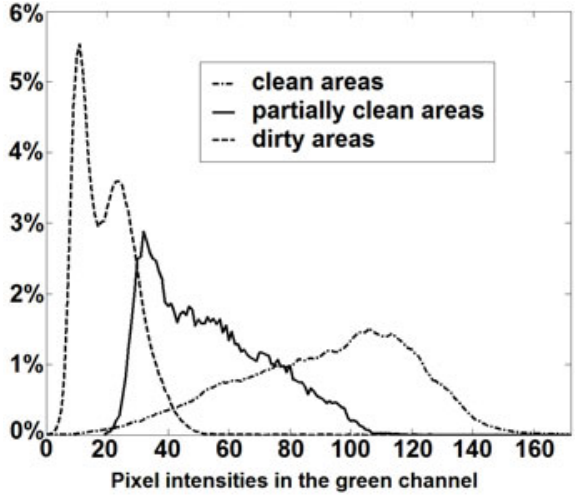

(g)

Fig. 2. (a)-(f) Intermediary stages of hand segmentation: (a) original image, (b) green channel image, (c) gradient of the green channel image, (d) initial detected contour, (e) adjusted contour, (f) extracted ROI (images (b)-(f) are shown in inverse colors); (g) Relative histogram of labeled data involved in the semi-supervised clustering algorithm
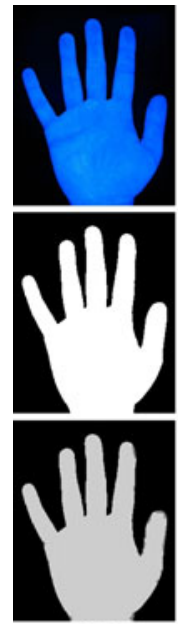

(a)
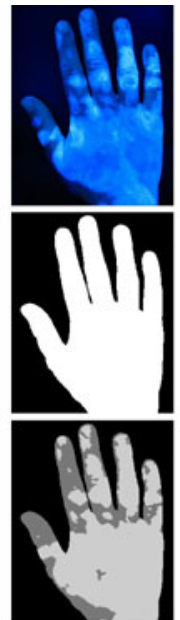

(b)
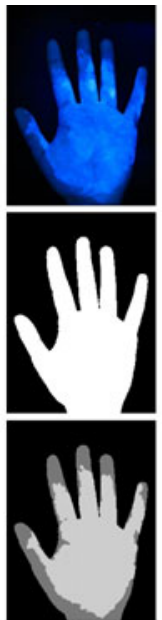

(c)
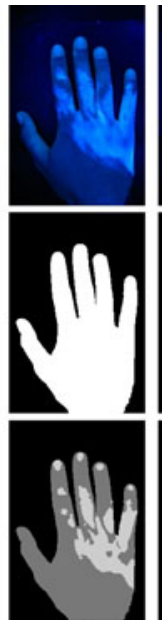

(d)
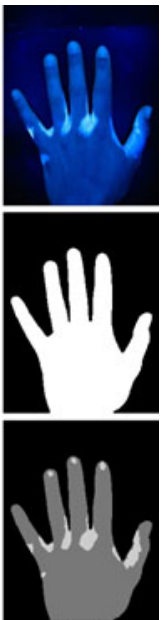

(e)

Fig. 3. Qualitative evaluation results for visual inspection: (a)-(e) hands with total or partial disinfection. In each column: upper row shows original color image, middle row indicates the extracted ROI, bottom row exhibits the segmented image.

\section{Results and Discussion}

To test the efficiency of the system, a trilateral study was conducted. Medical workers including surgeons, nurses, students and supporting staff from different 
Table 1. Quantitative results regarding the clean areas of the hands from Fig. 3

\begin{tabular}{|l|c|c|c|c|c|}
\hline Hand & $(\mathrm{a})$ & $(\mathrm{b})$ & $(\mathrm{c})$ & $(\mathrm{d})$ & $(\mathrm{e})$ \\
\hline Before compensation $(\pi)$ & $98.72 \%$ & $71.37 \%$ & $72.12 \%$ & $24.62 \%$ & $8.73 \%$ \\
\hline After compensation $\left(\pi_{w}\right)$ & $99.47 \%$ & $73.90 \%$ & $75.90 \%$ & $25.73 \%$ & $8.41 \%$ \\
\hline
\end{tabular}

hospitals volunteered to take part in the trials. Institutes were selected from Budapest (Hungary), Tîrgu-Mureş (Romania) and Singapore.

In an initial phase, approximately 200 images were recorded from dozens of different subjects, intentionally having varying disinfected area ranging from $0 \%$ to $100 \%$, forming a generalized teaching set.

Detected boundaries underwent a double investigation: first a visual inspection was applied, followed by an automatic evaluation of the pixel intensities in the immediate neighborhood of the detected boundary. This latter criterion approved the boundary when the great majority (e.g. 90\%) of inner neighbor pixels had higher intensity (in the green channel of the original image) than the great majority of outer neighbor pixels. In $99.2 \%$ of the tested cases, the boundary of the hand was correctly identified. The intermediary and final result of a successful case are presented in Fig. 2(a)-(f). The labeled data for supervised clustering was manually selected by experts from a learning data set that consisted of 64 different images. The relative histogram of labeled pixels belonging to the three classes is shown in Fig. 2( $(\mathrm{g})$. The three classes have significant overlapping parts.

The algorithm was tested on several images with different supervision rates $(\sigma)$ ranging between 0.005 and 0.03 , and the optimal value of $\sigma$ was derives to be 0.015 . Fig. 3 exhibits the result of five successful segmentations; identified clean areas are drawn in lighter gray, while dirty parts appear in darker gray. Table 1 gives the results of the quantitative evaluation of the same five images. As a guideline for future applications, we consider a hand appropriately disinfected, when it resembles the case shown in Fig. 3(a), having the final estimated percentage greater than $98 \%$.

During the test of the system, one of the challenges experienced was to account for the skin differences. Using calibrated image recording conditions (like foxed exposure time, ISO value of the camera) and including labeled data into the clustering algorithm resolved this problem with a remarkable accuracy.

The proposed image processing algorithm was implemented in $\mathrm{C}++$ programming language, using Intel's OpenCV computer vision libraries. Image processing tasks rarely handle individual pixels, most operations apply to the whole image (possibly restricted by a ROI), thus facilitating parallel execution. This enables us to provide the final evaluation results of an image in less than 1.5 seconds.

\section{Conclusions}

Since insufficient sterility continues to cause serious problems in health care around the globe, we exerted significant efforts to support the prevention of Hospital-Acquired Infections. We have built a mobile device that can perform the 
objective evaluation of the hand's disinfection ratio. The equipment is intended to help surgeons and hospital staff to reduce HAI rates, through teaching better hand hygiene practice. We use a UV reflective powder mixed to the regular alcoholic hand rub, and then clean areas of the hand are identified from digital images taken under UV lighting. The main contribution was the creation of an automated device and adjoint procedure that efficiently and accurately segments the digital image of the hand, and then classifies the treated areas using a semisupervised fuzzy $c$-means clustering algorithm. The performance of the system was evaluated on a large set of images, leading to an overall accuracy of $98.5 \%$, as verified by a human professional. It has been shown in trilateral clinical trials that the system is qualified for prevailing the omitted parts of the hand after hand washing. Meanwhile, it does not significantly disrupt the regular medical workflow, and is also capable of tracking disobedience to hand hygiene protocol, such as wearing jewelry or artificial nails.

\section{References}

1. European Center for Disease Prevention and Control: Annual Epidemiological Report 2008: Focus on Healthcare-associated infections, special chapter, pp. 16-38 (2008)

2. Pollack, A.: Rising threat of infections unfazed by antibiotics, New York Times (February 26, 2010)

3. Plowman, R., Graves, N., Griffin, M., Roberts, J.A., Swan, A.V., Cookson, B., Taylor, L.: The socio-economic burden of hospital acquired infection. Public Health Laboratory Service, London (1999)

4. Korniewicz, D.M.: Hand hygiene compliance and healthcare-associated infectionsWho is responsible? Touch Briefings-Infection Control, 37-39 (2007)

5. Xhale Technologies Inc.: HyGreen, http://www.xhale.com/hygreen

6. Girou, E., Loyeau, S., Legrand, P., Oppein, F., Brun-Buisson, C.: Efficacy of handrubbing with alcohol based solution versus standard handwashing with antiseptic soap: randomised clinical trial. Brit. Med. J. 325, 362-365 (2002)

7. Goroncy-Bermes, P.: Hand disinfection according to the European Standard EN 1500 (hygienic handrub): a study with Gram-negative and Gram-positive test organisms. Int. J. Hyg. Environ. Health 204(2-3), 123-126 (2001)

8. Boyce, J.M., Pittet, D.: Guideline for hand hygiene in healthcare settings: recommendations of the HICP Advisory Committee and HICPAC/SHEA/APIC/IDSA Hand Hygiene Task Force. Morbidity\&Mortality 51(RR16), 1-44 (2002)

9. CDC Joint Commission: Measuring hand hygiene adherence: overcoming the challenges. Oakbrook Terrace, IL (2009)

10. Pittet, D., Mourouga, P., Perneger, T.V.: Compliance with handwashing in a teaching hospital. Ann. Inter. Med. 130(2), 126-130 (2002)

11. WHO Collaborating Centre for Patient Safety Solutions: Improved hand hygiene to prevent health care-associated infections. Patient Safety Solutions 1(9), 1-4 (2007)

12. Szilágyi, L., Benyó, Z., Szilágyi, S.M., Adam, H.S.: MR brain image segmentation using an enhanced fuzzy $c$-means algorithm. In: 25th Annual Int'l. Conference of IEEE Engineering in Medicine and Biology Society, pp. 724-726 (2003)

13. Bezdek, J.C.: Pattern recognition with fuzzy objective function algorithms. Plenum, NY (1981) 